\title{
Heuristic Acquisition for Data Science
}

\section{Editorial for Special Issue of Information Systems Frontiers}

\author{
Lydia Bouzar-Benlabiod $^{1}$ - Stuart H. Rubin ${ }^{2}$ \\ Published online: 2 September 2020 \\ (C) This is a U.S. Government work and not under copyright protection in the US; foreign copyright protection may apply 2020
}

\section{Introduction}

The Traveling Salesman Problem (TSP) was first formulated in 1930 and is one of the most studied problems in optimization. If the optimal solution to the TSP can be found in polynomial time, it would then follow that every NP-hard problem could be solved in polynomial time, proving $\mathrm{P}=\mathrm{NP}$. We demonstrated an algorithm, which finds $\mathrm{P} \sim \mathrm{NP}$ with scale, Rubin et al. 2016, 2018. Using a $\delta-\varepsilon$ proof, it is straightforward to show that as the number of cities goes to infinity, $\mathrm{P}$ goes to NP (i.e., $\delta>0$ ). This was demonstrated using a quadratic number of parallel processors because that speedup, by definition, is polynomial. A fastest parallel algorithm was defined. Using an arbitrary run of 5000 cities, we obtained a tour within $0.00001063 \%$ of the optimal using 4,166,667 virtual processors (Intel Xenon E5-1603 @ $2.8 \mathrm{GHz}$ ). To save the calculated extra 209 miles would take a quantum computer over $(5000 ! /(2 * * 4978 * 22 !)) * 267,782$ centuries. Clearly, the automated acquisition of heuristics and the associated $\mathrm{P} \sim \mathrm{NP}$ solutions are an important problem warranting attention. Machine learning through self-randomization was demonstrated in the solution of the TSP.

It is becoming increasingly rare for software to run in isolated environments. Rather, the software of the future will need to support government and industry in distributed heterogeneous computing environments connected by secure networks. Ensuring the quality of that software provides information dominance.

Stuart H. Rubin

stuart.rubin@navy.mil

Lydia Bouzar-Benlabiod

1_bouzar@esi.dz

1 Laboratoire de Communication des Systèmes Informatiques (LCSI), Ecole Nationale Supérieure d'Informatique (ESI, Algeria), Oued Smar, Algeria

2 Naval Information Warfare Center (NIWC) Pacific, Intelligent Sensing Branch, San Diego, CA, USA
It follows that the automatic programming (software automation) of quality software is a necessary objective towards achieving information dominance. Randomization can provide the means by which the human on the loop realizes a high-level specification in effective code. Conventional compilers are essentially context-free translators. They are thus realizable through the use of context-free grammars with the translation of a few context-sensitive features through the use of attribute grammars. Nevertheless, progress in automatic programming hinges on the use of context-sensitive specification languages (e.g., structured English). Such languages are effectively compiled through the actions of rule bases. In fact, the first compilers were rule-based.

The design of rule-based compilers is not a theoretical problem. Rather, it is an economical process for the acquisition of knowledge for these compilers that has evaded solution to date. It follows that the solution of the automatic programming problem, including attendant cybersecurity, can be reduced to the efficient and cost-effective solution of the knowledge acquisition bottleneck. However, the more complex the knowledge, the more its' efficient acquisition depends on the acquisition of a salient knowledge base. This (second) knowledge base serves as heuristics for guiding the acquisition of the first one.

If knowledge did not beget knowledge, there would be no need for heuristics, which was shown early on by Mitchell et al. 1986, for Dendral and Meta-Dendral, to not be the case. It logically follows that the focal problem here is how to apply knowledge-based systems to their knowledge-acquisition bottleneck. The solution of this problem necessarily entails selfreference with scale (e.g., daisy-chained self-reference). A practical implication here is that secure networks will play a critical role in facilitating daisy-chained self-reference.

Self-reference implies the use of heuristics to prevent inherent incompleteness, Kfoury et al. 1982; Uspenskii 1987. The science of randomization offers a new way forward in the application of knowledge bases to themselves in order to extend them. For a real-world example, consider the following set of five rules pertaining to the solution of the TSP (Fig. 1). 
R1: Visit the closest city on Open, resolving ties arbitrarily, and remove it to Closed. (Closed is used to ensure that no city may be visited twice.) Increase the tour length by the distance to this city.

R2: Traverse to the closest city on Open and then to the closest city to it on Open. No city may be visited twice. Compute all path lengths. Visit cities on the shortest path length, resolving ties arbitrarily, and remove them to Closed. Increase the tour (length) by the shortest path (length).

R3 (heuristic pruning): If the search-ply, at any city, exceeds t, execute R1.

R4: If no city remains on Open, return to the starting city and exit with the found solution.

R5: Run rule base with $t=1,2,4, \ldots, 2^{* *} \mathrm{n}$, fitting a curve using the Newton Forward Difference Formula until the predicted runtime for $t$ exceeds the allowance.

Fig. 1 A simple rule base for a heuristic solution of the TSP

Rules are self-contained coherent algorithms containing one or more entrance preconditions, a coherent action, and one or more exit post-conditions, which are often used to update a blackboard. Next, R1, R3, and R4 may be applied to $\mathrm{R} 2$ to randomize the rule base and in so doing extend its capability for finding a best heuristic solution. Here is the selfreferentially randomized rule base, consisting of just two rules (Fig. 2). It is more general (capable) than the base from which it was derived. Appeal is made to Church's Thesis, Kfoury et al. 1982, to show that the mechanics of self-referential randomization are effectively realizable. These mechanics can be extended by inductive and abductive discovery through the use of schema-based search and I/O constraints, in Rubin and Bouabana-Tebibel (2016). The implications for cybersecure automatic programming follow from the previous discussion.

It follows from previous results that automatic programming and hence knowledge acquisition is an NP-hard problem. It also follows that the quadratic-time solution to this problem entails the use of heuristics. Furthermore, it has been empirically demonstrated that self-reference is the key to successful heuristic acquisition, based on transferring results for the n-puzzle, Rubin et al. (2015). However, the results shown above make it clear that while this works, it can be arbitrarily difficult to acquire a set of appropriate heuristics. It then follows that the search for such heuristics must be pruned through the application of appropriate knowledge, which is to state that self-reference is key.

The need for search heuristics is inherent. This follows because, like the TSP, automatic programming is NP-hard. Even a full-scale programmable quantum computer could do no better than reduce the search by $O\left(2^{* *} \mathrm{n}\right)$. The TSP, as previously discussed, is $O(\mathrm{n} !)$, where $O(\mathrm{n} !)>>O(2 * * \mathrm{n})$. This differential is even more pronounced for true automatic programming, which is as complex as $O(\mathrm{n} * * \mathrm{n})$, where $O(\mathrm{n} * * \mathrm{n})>>O(\mathrm{n} !)$. Heuristics, heuristic acquisition, and heuristic transference are inherently better enablers of machine learning through self-randomization than can quantum computers be, Rubin et al. (2015). However, this is not to be interpreted as an argument to preclude the development of quantum computers to the extent possible. Rather, it is meant to put priorities in perspective.

Clearly, this self-randomization of a knowledge base cannot be limited to any domain-specific set of problems. It is evidently more general than that. If the domain is set to that of any type of knowledge-based software associate (i.e., software whose purpose is to help the programmer in creating software - including itself), then cyber-secure automatic programming will likewise benefit. This follows because if any NP-hard problem (e.g., the TSP) can be shown to benefit from the approach, it then follows that every NP-hard problem will likewise benefit (e.g., even the very NP-hard problem of automatic programming).

It follows from the results of Rubin 2007 that knowledge can grow to be of unbounded density by process of generalization. Maximizing the quantity of applicable knowledge can be shown to minimize the mean time for discovery while maximizing the mean quality of the knowledge, which may be discovered.

The attainable density of knowledge is also dependent upon its representation (e.g., the missionaries and cannibals problem), Amarel 1968. Translating the representation of knowledge, in general, requires search and control knowledge to constrain that search. It follows that search, 
R1: While Open $<>\{\}$

Begin

While the search-ply $\leq \mathrm{t}$

Begin

Traverse to the closest non-visited city on Open.

Resolve ties arbitrarily.

Increase the path length by the distance to this city.

Visited cities in a loop are treated the same as those on Closed.

End

Removed visited cities, on the minimal path length, to Closed. (Closed is used to ensure that no city may be visited twice.)

Resolve ties arbitrarily.

Increase the tour (length) by this path (length).

End

Exit with the solution.

R2: Run rule base with $\mathrm{t}=1,2,4, \ldots, 2^{* *} \mathrm{n}$, fitting a curve using the Newton Forward Difference Formula (interpolating polynomial) until the predicted runtime for t exceeds the allowance.

Fig. 2 A more general self-randomized rule base

self-reference, and randomization are inherent to automatic programming in the large. The result is that automation, and hence intelligence, derives from effective very-high level representations (e.g., natural language) of contextsensitive knowledge.

This is not knowledge acquisition as portrayed in the literature. Rather, it takes the view that candidate knowledge stems from abductive questions that ask, "What would have to be true to obtain a further state randomization?" For example, helium balloons rise and hydrogen balloons rise. What would have to be true for gasses less dense than air to cause a balloon to rise? The answer is that hot air balloons must rise. Conventional expert system shells do not have access to this knowledge, unless it is literally supplied. Here, one sees how self-referential randomization can be driven by questionasking and/or experiment - leading to the creation of new and valid knowledge, which can be quite different from anything in the knowledge base(s), but which may be abduced from it.
Figure 2 demonstrates that the process of self-randomizing a rule base can be quite arduous and thus require an economy of scale to be effective. Nevertheless, there are inductive logics of abduction, which can be quite effective for selfrandomization - even in the small. One such inductive logic of abduction involves the normalization and randomization of property lists.

One of the problems with property sets is that combinations of variables are, in general, what is enabling generalization, which involves $n$ choose $r$ possibilities - exponential. Thus, while it is relatively simple (and eminently practical) to induce that lighter than air gasses are what causes a balloon to rise, it is far more arduous to determine say what are the properties of $\mathrm{TiO} 2$ that enable it to crack water in the presence of sunlight (i.e., in the search for better catalysts). Clearly, the only hedge against this inherent combinatorics problem is knowledge; and, the best way to acquire such knowledge is through selfrandomization. 
Biermann (1985) wrote a non-creative expert system, at Duke University, that synthesized "impressive" LISP programs using rules. The main inefficiency was the knowledge-acquisition bottleneck, or rule-discovery process. Self-randomizing rule bases can mitigate that bottleneck and enable the creation of cyber-secure synthetic software. This software can be represented in the form of an expert system running coherent rules, or in the form of functional programs. The point is that not only are all manner of software associates possible, but the high-level codes themselves can also be synthesized with the human on the loop, which again follows from the work of Biermann (1985).

Self-randomizing knowledge bases define more intelligent systems. These knowledge repositories will drive software associates and synthesizers, where the larger the scale, the more capable the system. Fortunately, a proof of concept is possible in the small. Eventually, such machines may become capable of unbounded state and representation randomization, Rubin 2007.

\section{The Special Issue}

The first paper (Șen et al. 2020) addresses the important business topic of predicting the credit worthiness of a requested small business loan. It combines support vector machines (SVM) with genetic algorithms to evolve the hyperplane for a better data fit by the SVM. The paper explains that problems, such as credit scoring, are at best inherently incomplete. This suggests the utility of applying heuristics and meta-heuristics towards finding the best fitting solution for the data space. Moreover, it implies that an area for future advancement is the application and development of machine learning technologies to the acquisition and refinement of heuristics. Indeed, even where data is available, many of these problems are NPhard (e.g., the TSP) - making the use of heuristic methods the only way for obtaining near-optimal solutions in tractable time. The advent of the quantum computer does not change that, since most of these problems are well-beyond the reach of theoretical quantum computations (e.g., the TSP, which is $O(\mathrm{n} !))$.

The second paper (Xu et al. 2020) introduces sentiment analysis into Principal Component Analysis for modeling the stock market using heuristic means-end analysis. The significance of this paper is that it addresses a domain, where there is an inherent need for heuristics. Heuristics may be thought of as an extension to fuzzy logic, where the need for precision is offset by inherent uncertainty in the application domain. As it turns out, this is the general case, rather than a specific exception.

The third paper (Kuko and Pourhomayoun 2020) addresses the problem of cervical cell cancer detection using ensemble and deep learning methods to automate the otherwise time- consuming Pap smear or LBC test using machine vision. Furthermore, Rubin has invented a new machine learning technology, which is based on randomization and is being commercialized to replace neural technologies. Indeed, we find that the human brain works more on the basis of randomization and has little to nothing in common with neural technologies.

The fourth paper (Tian et al. 2020) addresses feature selection for a Convolutional Neural Network (CNN) through the use of transfer learning. Transfer learning is proposed using an evolutionary programming framework for learning the features as well as the overarching model. An open question remains; namely, tractable evolution when the distance between the source and target domains is significant. It is here that the need for salient heuristics is greatest (see the second paper). The editors believe that much progress remains to be made in the selection and evolution of heuristics. This paper assists by expounding upon that predilection.

The fifth paper (Smiti and Soui 2020) addresses the important problem of bankruptcy prediction using deep learning to build a predictive model. K-means as well as a support vector machine are fused to yield the final approach. This is an ongoing area of interest to financial lenders. It inherently requires feature extraction as well as heuristic processing to improve the model-building process. Thus, it should likewise benefit from the inclusion of evolutionary programming. Clearly, self-organizing machines, such as this, require knowledge to kickstart them. The open question pertains to the acquisition and application of (self-referential) salient knowledge.

The sixth paper (Manouchehri et al. 2020) models data using a Dirichlet distribution for Bayesian statistical inference combined with a variational calculus for estimating model parameters and the resultant complexity. Models address the categorization of image spam, diabetes mellitus, and the detection of infectious hepatitis - all of great practical value. Results suggest that heuristic modeling, like all other modeling, needs to be jump started using domain-specific constructs pertaining to features, their statistical distributions, and mutual dependencies. Thus, it may be the case that modeling languages need to represent what we know and constrain the space of what we need to know to effectively evolve everbetter heuristics for use in a complex world. One needs heuristics to acquire knowledge and knowledge to constrain search-control heuristics. These topics lie at the heart of information reuse, integration, and data science.

The seventh paper (Kafle et al. 2020) deals with the important problem of knowledge representation in knowledge bases for assisting neural networks in understanding natural language. It used to be held that recurrent neural networks (RNNs)could learn to understand natural language; but this approach has recently yielded to model-based approaches. The problem is that RNNs do not scale and do not model their 
inputs. Knowledge must have multiple representations if it is to be reused in distinct domains. We see that transfer learning necessarily goes beyond the rules to encompass the representation of those rules. An open question is whether representational search is more efficacious or search to fit a neural model. In as much as both forms of search are generally NP-hard problems, it may be that cooperation between the two forms is best, which is something that this paper put to the test.

The eighth and final paper (Johnson and Khoshgoftaar 2020), deals with training predictive models with classimbalanced data. The problem is particularly acute in attempts to train hidden-layer neural networks with big data. Here, unless sets of randomizing features are available, the data needs to be sampled so as to render its use in training neural nets tractable without losing its characterization. The paper addresses these interrelated issues. However, in so doing, it raises the question if neural networks are a proper modeling tool for the majority of domains, since error correction, in their training, leads to intractability - unlike the case for humans say.

\section{Future Research}

The In this section, an introductory heuristic calculus is defined and used to illustrate the mechanics for the scalable randomization of case-based knowledge by process of transformative analogy. We will demonstrate how the heuristic calculus can augment the predicate calculus to reason about undecidable predicates as well as to speedup otherwise intractable resolutions. First, consider a simple problem in the second-order predicate calculus involving search control; namely,

$\exists \mathrm{x}$ Boy $(\mathrm{x}) \rightarrow$ Likes $(\mathrm{x}$, ice cream)

$\forall \mathrm{x}$ Boy $(\mathrm{x}) \wedge$ Weather $($ hot $) \rightarrow$ Likes $(\mathrm{x}$, ice cream $)$

$\forall \mathrm{y}$ Girl $(\mathrm{y}) \wedge$ Weather $($ hot $) \rightarrow$ Likes $(\mathrm{y}$, ice cream)

$\exists$ z Likes $(z$, candy) $\rightarrow$ Likes $(z$, ice cream)

By the composition of (1) and (4) we obtain:

$\exists$ z Boy $(z) \vee \exists z$ Likes $(z$, candy $) \rightarrow$ Likes $(z$, ice cream $)$

That is, there exists someone who is a boy, or likes candy, and thus likes ice cream.

Similarly, by the composition of (3) and (4) we obtain:

$\forall \mathrm{z}$ Girl $(\mathrm{z}) \wedge$ Weather $($ hot $) \vee \exists z$ Likes $(\mathrm{z}$, candy $) \rightarrow$ Likes $(\mathrm{z}$, ice cream)

We want to know if there exists a girl who likes ice cream in the absence of hot weather. Notice that just because someone likes candy does not ensure that they are a girl. Clearly, there is no way to deduce the requested information using the second-order predicate calculus here. Equating the right-hand sides of (1) and (2) yields,

$\exists \mathrm{x}$ Boy $(\mathrm{x}) \leftrightarrow \forall \mathrm{x}$ Boy $(\mathrm{x}) \wedge$ Weather (hot) $\mid$ Likes (x, ice cream)

Substituting $\mathrm{z}$ for $\mathrm{x}, \mathrm{y}$; equating the right-hand sides of (2) and (4); and, substituting results into (7) yields,

$\exists \mathrm{z}$ Boy $(\mathrm{z}) \leftrightarrow \forall \mathrm{z}$ Boy $(\mathrm{z}) \wedge$ Weather $($ hot $) \leftrightarrow \forall \mathrm{z}$ Girl $(\mathrm{z}) \wedge$ Weather

(hot) | Likes (z, ice cream)

It then heuristically follows that the universal qualifier in (8) can be replaced by the existential qualifier,

$\exists \mathrm{z}$ Boy $(\mathrm{z}) \leftrightarrow \exists \mathrm{z}$ Girl $(\mathrm{z}) \mid$ Likes $(\mathrm{z}$, ice cream)

Weather (hot) was removed for boys and it can be removed for girls because it shares the same right-hand side; namely, Likes ( $\mathrm{z}$, ice cream) and because the pattern of changing a universal qualifier to an existential qualifier can be followed for the girls - just like for the boys. Thus, (6) may be rewritten as,

$\exists z$ Girl $(z) \vee \exists z$ Likes $(z$, candy $) \rightarrow$ Likes $(z$, ice cream $)$

Equation (10) makes it clear that it is highly likely, but not assured, that there exists a girl who likes ice cream in the absence of hot weather. It is interesting to note that eq. (10) reflects this uncertainty, since it is never necessary, strictly speaking, for the first predicate to be satisfied to invoke the implication.

Just as (1), (2), and (3) can be combined, it is also possible to equate non deterministic left-hand sides in the manner just illustrated. Moreover, instances may be proper subsets and we may also introduce $\Psi, \mathrm{E}$ for "for most" and "for some", respectively. In the previous example, instead of replacing $\forall$ with $\exists$, we could have developed it to replace $\Psi$ with $\mathrm{E}$ for a somewhat more general result. In addition, compositions may find practical use. Possibilities may be computed using a calculation proportional to the number of derivational paths (i.e., one was shown here). Finally, whenever there is an error, the correct resulting equation must be acquired to correct the inductive mechanism by way of eliminating the need for inferential reasoning here.

It should be practical to establish rules of inference for the heuristic calculus just as has been done for the predicate calculus, using different, albeit more complex, transformation rules. The heuristic calculus is not subject to essential incompleteness (Uspenskii 1987) and need not ever be intractable because heuristics can be applied to speed up inferences as well as resolutions - so long as a slight loss of validity is acceptable. This follows because heuristic transformations are not necessarily validity preserving - particularly when applied to a general domain. Next, an example of the heuristic calculus, as applied to non-deterministic reasoning, will be provided. 
Many processes are inherently stochastic. Here, there may be more than one correct answer and any attempt to reduce them to a single correct answer is done strictly as a matter of convenience. Consider a move in chess, which is similar to battle management, where say a Knight forks the pair of opponent Rooks, where necessarily limited look-ahead cannot resolve which Rook, if any, to take. W represents white and B represents black.

$\neg$ Check (WKing) $\wedge$ Fork (BRook1, BRook2) $\rightarrow$ Search

(Take (BRook1), Take (BRook2))

Search $(($ Take (BRook1), Take (BRook2)) $\rightarrow$ Take (BRook1)

Search $(($ Take $($ BRook1), Take (BRook2)) $\rightarrow$ Take (BRook2)

Equations (12) and (13) are non-deterministic as sometimes limited or even heuristic search cannot resolve between two or more actions. Here, both are proper.

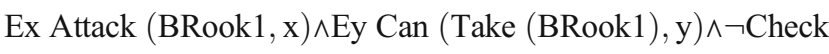

$$
\text { (WKing) } \rightarrow \text { Take (BRook1) }
$$

This says that if the black Rook can attack some pieces and some of our pieces can take the black Rook and our King is not in check, then take the first black Rook. Of course, there are other concerns in a real chess game, such as the relative point value of all exchanged pieces, the potential setup for a checkmate, and the like. These will be ignored here for the sake of simplicity of exposition.

Equating the left-hand sides of (12) and (13), we obtain,

Search ((Take (BRook1), Take (BRook2)) |Take (BRook1) $\leftrightarrow$ Take (BRook2)

Here, the contextual match is a little more difficult to achieve than in our previous example. That is,

Search (Take (BRooki)) cSearch ((Take (BRook1), Take (BRook2))

Proper subsets are defined to satisfy their contextual requirements. Substituting (15) into (14) twice yields,

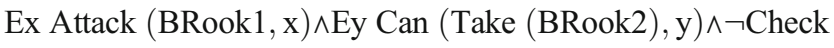

$$
\text { (WKing) } \rightarrow \text { Take (BRook2) }
$$

At first glance, this new heuristic rule, which is not deducible on the basis of the given knowledge, is atypical. That is, one would have expected to also see, Ex Attack (BRook2, x), but this was not pattern matched and hence remains unaltered. Basically, the induced rule says something to the effect, "If your first black Rook attacks me and I'm not in check, I can and will take your second black Rook." This makes for an interesting game. If we wanted to also induce a heuristic rule to counter the imminent attack by the first black Rook, we could include the following pair of simple rules.

Ex Attack (BRook1, x) $\rightarrow$ Take (BRooki)

Ex Attack (BRook2, x) $\rightarrow$ Take (BRooki)

Here, it is understood that,

Take (BRooki) $\in\{$ Take (BRook1), Take (BRook2)\}

Thus,

Ex Attack (BRook1,x)↔Ex Attack (BRook2, x) | Take (BRooki)

Applying (21) to (17) gives the result:

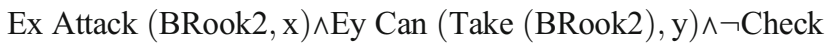

$$
\text { (WKing) } \rightarrow \text { Take (BRook2) }
$$

Errors of transformation are caught through the use of constraint checking. This does not imply that everything that passes constraint checking is valid. Rather, passing the constraint checker simply ensures that it is logical for the scope of recorded information. Since this information is potentially infinite, it follows that while the results of heuristic inference are far more likely to be valid than not, again validity is not assured.

A heuristic calculus is potentially far more complex than the predicate calculus, but all of this additional complexity stems from heuristic inference, constraint checking, and related search. The heuristic nature of this search makes it possible to fuzzify the predicate calculus by replacing $\forall$ with $\Psi$ (i.e., for most) and $\exists$ with $E$ (i.e., for some). In fact, the heuristic calculus can be defined as a rule-based mechanics, where corrective heuristic resolutions may be incrementally acquired. This can ensure the generality of the predicate calculus combined with the domain-specificity (including the tractability possible in an expert system, which defines a domain-general mechanics). Moreover, heuristic calculi need never suffer from essential incompleteness (Uspenskii 1987).

Fuzzy logic (Zadeh 1996) and even computing with words (Pedrycz and Rubin 2010) does not and cannot capture that, 
which the heuristic calculus can. Heuristic and fuzzy qualitative learning are the underpinning reasons for this differential.

Moreover, the heuristic calculus can be used to circumvent the intractability of resolution theorem proving - not only by heuristically resolving the back-cut mechanism of Prolog (Nilsson 1980), but through the acquisition of heuristics, which can do more for speedup than say granularization alone. But the main benefit is an attendant capability for commonsense reasoning, which is uniquely provided by heuristic acquisition.

Reviewers We would like to thank the reviewers (listed below) for their precious help.

Miloud Bennama; Lamia Berkani; Sidney Chapman; Asmaa Chebba; Simon Fong; Hamid Haddadou; Leila Hamdad; Walid Khaled Hidouci; Abdellah Kaci; Selma Khouri; Tamas Kristof; Lynda Said L'Hadj; Katja Langenbucher; Huimin Lu; António Neves; Houda Oufaida; Yahya Slimani; and, Lamia Yessad.

\section{References}

Amarel, S. (1968). On representations of problems of reasoning about actions. Mach Intelligence, 3, 131-171.

Biermann, A.W. (1985). Automatic programming: A tutorial on formal methodologies. J Symbolic Computation, 1 (2).

Johnson, J. M., \& Khoshgoftaar, T. M. (2020). The effects of data sampling with deep learning and highly imbalanced big data. Information Systems Frontiers, 22(5). https://doi.org/10.1007/ s10796-020-10022-7.

Kafle, S., de Silva, N., \& Dou, D. (2020). An overview of utilizing knowledge bases in neural networks for question answering. Information Systems Frontiers, 22(5). https://doi.org/10.1007/ s10796-020-10035-2.

Kfoury, A. J., Moll, R. N., \& Arbib, M. A. (1982). (1982). A programming approach to computability. Ne w York: Springer-Verlag Inc..

Kuko, M., \& Pourhomayoun, M. (2020). Single and clustered cervical cell classification with ensemble and deep learning methods. Information Systems Frontiers, 22(5). https://doi.org/10.1007/ s10796-020-10028-1.

Manouchehri, N., Nguyen, H., Koochemeshkian, P., Bouguila, N., \& Fan, W. (2020). Online Variational learning of Dirichlet process mixtures of scaled Dirichlet distributions. Information Systems Frontiers, 22(5). https://doi.org/10.1007/s10796-020-10027-2.

Mitchell, T. M., Carbonell, J. G., \& Michalski, R. S. (1986). Machine learning: A guide to current research. NY, NY: Springer-Verlag Inc..

Nilsson, N. J. (1980). Principles of artificial intelligence. Mountain View: Morgan Kaufmann Publishers, Inc..

Pedrycz, W., \& Rubin, S. H. (2010). Data compactification and computing with words. Int Sci J Engineering Applications of Artificial Intelligence, 23, 346-356.

Rubin, S. H. (2007). On randomization and discovery. Information Sciences, 177(1), 170-191.

Rubin, S.H. and Bouabana-Tebibel, T. (2016). NNCS: Randomization and informed search for novel naval cyber strategies, in Recent Advances in Computational Intelligence in Defense and Security, Studies in Computational Intelligence, R. Abielmona et al. (eds.) vol. 621, Springer International Publishing, Switzerland, pp. 193223.
Rubin, S.H. et al. (2015). On heuristic randomization and reuse as an enabler of domain transference, Proc. 2015 IEEE Intern. Conf. on Info. Reuse and Integr. (IRI), San Francisco, CA pp. 411-418.

Rubin, S.H., Bouabana-Tebibel, T., Hoadjli, Y., and Ghalem, Z. (2016). Reusing the NP-hard traveling-salesman problem to demonstrate that P NP, Proc. 2016 IEEE International Conference on Information Reuse and Integration (IRI), Pittsburgh, PA, 28-30 Jul. 2016, pp. 574-581.

Rubin, S.H., Bouabana-Tebibel, T., Grefe, W.K., Hoadjli, Y., and Ghalem, Z. (2018). On the tractable acquisition of heuristics for software synthesis demonstrating that $\mathrm{P} \sim \mathrm{NP}$, quality software through reuse and integration, Advances in Intelligent Systems and Computing, Springer International Publishing, Switzerland (AISC 561), Ch. 1, pp. 1-23.

Șen, D., Dönmez, C. Ç., \& Yıldırım, U. M. (2020). A hybrid bi-level metaheuristic for credit scoring. Information Systems Frontiers, 22(5). https://doi.org/10.1007/s10796-020-10037-0.

Smiti, S., \& Soui, M. (2020). Bankruptcy prediction using deep learning approach based on borderline SMOTE. Information Systems Frontiers, 22(5). https://doi.org/10.1007/s10796-020-10031-6.

Tian, H., Chen, S., \& Shyu, M. (2020). Evolutionary programming based deep learning feature selection and network construction for visual data classification. Information Systems Frontiers, 22(5). https://doi. org/10.1007/s10796-020-10023-6.

Uspenskii, V.A. (1987). Gödel's incompleteness theorem, Translated from Russian. Moscow: Ves Mir Publishers.

Xu, Q., Chang, V., \& Hsu, C. (2020). Event study and principal component analysis based on sentiment analysis - A combined methodology to study the stock market with an empirical study. Information Systems Frontiers, 22(5). https://doi.org/10.1007/s10796-02010024-5.

Zadeh, L. A. (1996). Fuzzy logic = computing with words. IEEE Transactions on Fuzzy Systems, 4(2), 103-111.

Publisher's Note Springer Nature remains neutral with regard to jurisdictional claims in published maps and institutional affiliations.

Lydia Bouzar-Benlabiod is an assistant professor at Laboratoire de Communication des Systèmes Informatiques (LCSI), Ecole Nationale Supérieure d'Informatique (ESI, Algeria). She received a $\mathrm{PhD}$ degree in Computer Science from ESI and from Université d'Artois (France) in 2015. She received a magisterial degree from ESI in 2010. She is a member of The International Society of Applied Intelligence. She has co-edited two books and a special issue of the ISF Springer journal with Dr. Stuart Rubin.

Stuart H. Rubin is a senior scientist at the Naval Information Warfare Center (NIWC) Pacific, Intelligent Sensing Branch, in San Diego. He is a Fellow of the IEEE, the Society for Information Reuse and Integration (SIRI), the National Academy of Inventors (NAI), and the American Association for the Advancement of Science (AAAS). Dr. Rubin has a $\mathrm{PhD}$ in Information Science from Lehigh University and a certificate for Embodied Intelligence from MIT. He was an ONT Post-Doctoral Fellow and a tenured professor of computer science at Central Michigan University (CMU). Dr. Rubin was an electronic engineer with the US Army Research Labs, Ft. Monmouth, NJ from 1980 to 1983. He worked on projects involving the application of AI technology to the Very HighSpeed Integrated Circuits (VHSIC) program for which he was awarded the US Government Certificate of Merit in 1987. He also received the Navy Award of Merit for Group Achievement in 2002. Dr. Rubin holds 42 professional U.S. patents and published over 335 refereed papers including five book chapters plus five books, and has received five publication awards. 